\title{
Evaluating Two Methods of Testing Residual Sanitizer Concentrations in Food Service Establishments
}

Pooja Kumar ${ }^{1}$, Helen Heacock ${ }^{2}$

1 Lead Author, B. Tech Student, School of Health Sciences, British Columbia Institute of Technology, 3700 Willingdon Ave, Burnaby

2 Supervisor, School of Health Sciences, British Columbia Institute of Technology, 3700 Willingdon Ave, Burnaby

\section{Abstract}

Objective: Utensils and tableware are food contact surfaces that have the potential to transmit disease-causing microorganisms if not washed, cleaned and sanitized correctly in a food service establishment. To prevent utensils and tableware from becoming vectors of disease, it is essential that operators and staff are able to adequately sanitize them and accurately test for it in a quick and convenient manner. It is also essential that Environmental Health officers are able to test whether adequate sanitization is occurring during their routine inspections. Currently there are no guidelines indicating the correct method of testing. Therefore, this study investigated two methods used to test residual sanitation concentration in a dishwasher. The purpose of this research was to determine if there is any difference in the two methods currently being used, and if so, which method is the more reliable one.

Methods: LaMotte Chlorine Test Strips were used to detect the levels of chlorine in a commercial dishwasher. The chemically treated strips were dipped onto a freshly wet and washed utensil and directly in the rinse water of a dishwasher.

Results: The difference in the mean of 60 samples from two independent groups was analyzed. Thirty samples were obtained from location one, the dishware l, and thirty samples were obtained from location two, the rinse water of the commercial dishwasher. The mean residual concentration was calculated and compared. The means demonstrated there is a significant difference $(p=0.035)$ between the two groups; the average residual concentration was lower for the dishware compared to the rinse water.

Conclusion: Dishware is a vector capable of transmitting disease causing microorganisms if not sanitized adequately. Thus, it is important to ensure that dishes in a food service establishment have been thoroughly sanitized. The testing of that requires a consistent and reliable method. It is safe to assume that testing on the dishware is the best course of action to err on the side of caution.

Key words: test strip, chemical dispenser, chlorine, low temperature dishwashers, sanitation, tableware, rinse water, chlorine 


\section{Introduction}

In a commercial kitchen warewashing is the process of cleaning utensils and tableware by physically removing the dirt with the aid of a detergent, rinsing them clean with water and then applying a method of sanitization on them. Sanitization is the second last step of warewashing, just before air-drying, and it is possibly the most important. It is the step/stage where utensils and tableware are treated with extremely high heat or chemicals to eliminate most disease-causing microorganisms (1) or reduce them to levels that are considered safe for human health.

Utensils and tableware are considered to be effectively sanitized once they have achieved a 5-log reduction of pathogenic microorganisms (2). From a public health perspective, removing $99.999 \%$ of microbial pathogens on food contact surfaces is the only way to ensure that these surfaces no longer pose a risk on/for human health (3).

An adequate sanitizer concentration is to be maintained in a chemical dishwasher during each cycle to ensure that the function of the dishwasher is being achieved. The amount of sanitizer being fed into the machine can be controlled via an adjustable valve. However, monitoring the feed levels alone is not sufficient when determining whether adequate sanitation was achieved, and therefore testing the residual sanitizer concentration is required. The residual concentration is tested for in parts per million (ppm) with chemical specific test papers. The test paper is dipped in the rinse water from the last cycle of the dishwasher or on the surface of the utensil while it is still wet. The purpose of this is to observe a color change; the strength in color gives an indication of concentration in ppm (4). The color on the test paper is to be compared with the standard found on the test kit/test tube to determine the concentration of the chemical.
Currently, the challenge that exists is there is no universally acceptable method of using a test strip to measure the residual concentration of the sanitizer. Should a test strip be placed right on the clean, and wet utensil as soon as it comes out of the dishwasher? Or, should the test strip be placed directly under the jets, where the final rinse water is held? Which procedure would get the most accurate reading? Currently, there is no provincial legislation or guideline indicating an optimal procedure.

The purpose of this study was to determine if the two different methods used to test the residual sanitizer concentration of a dishwasher cycle in a food service establishment produce similar or different readings. Generally, Environmental Health Officers (EHOs) and restaurant staff use the surface of the dish or the rinse water to measure whether adequate sanitization was achieved. Currently, the BC Food Premises Regulation (1) states no universal method to accurately measure, nor does the BC Food Services and Retail Code (2).

\section{Evidence Review}

\section{a. Importance of sanitizing utensils}

One of the top five risk factors for contaminating food is the inadequate sanitization of kitchen utensils (5). Furthermore, contaminated equipment is one of the top three risk factors responsible for foodborne illnesses acquired at a retail food service (5). After examining British Columbia's legislation pertaining to public health and, in particular, food service establishments, a standard test for measuring residual sanitizer concentration after a warewashing cycle does not exist, nor what the frequency of testing should be. When conducting this test during a 
routine inspection, EHOs do not have a specific and accepted method to follow. Conflicts can arise when a food service establishment operator does not agree with the way the EHO is conducting his or her inspection. It can be challenging for EHOs to get operator compliance when there is no legislation to refer to. Not only can it cause confusion between EHOs and operators, it can compromise the health of the public if it is unknown whether adequate sanitization has been achieved, which is essential in eliminating potential foodborne illnesses.

If these issues can be resolved with the simple implementation of a policy or a change in a regulation, and they can benefit public health, then policies or regulations should be implemented.

\section{b. Methods of warewashing - manual and mechanical}

Manual warewashing is accomplished by using a three-compartment sink. Each step - washing, cleaning and sanitizing - in that order, is carried out in an individual compartment (2). It is important to rinse and remove all organic matter before the actual sanitization occurs to get the most effective results (6). Sanitation can be achieved via two ways - thermal or chemical. Heat is rarely used in manual dishwashing because it is a health hazard to submerse a hand in water that is at least 77 ${ }^{\circ} \mathrm{C}$, the required temperature for to achieve sanitization. For that reason, chemical sanitation is the preferred method in manual dishwashing (7).

The mechanical method of warewashing is achieved by using a commercial dishwasher. Utensils must be scraped and pre rinsed before being placed onto the rack as a dishwasher only performs a wash cycle and a rinse cycle. In the wash cycle, detergent and hot water are applied to the utensils; in the rinse cycle, sanitizer is applied to the utensils and then they are given a final rinse (8).

Hobart is the manufacturer that supplies most of the commercial dishwashers to food service establishments (9). Commercial dishwashers can come in two forms - high temperature or low temperature. Almost all the steps are the same for both types, the only difference is the final sanitization stage.

High temperature dishwashers use heat to sanitize whereas low temperature dishwashers use chemicals to sanitize. In a high temperature dishwasher, time, temperature and pressure are key variables. The surface of the dish must reach a temperature of $71^{\circ} \mathrm{C}$. This can be measured in a few different ways including max recording thermometers (dishwasher thermometers), thermolabels and infrared temperature guns (8). In a low temperature dishwasher, sanitization is achieved via chemicals. The chemicals used are sodium hypochlorite (chlorine), quaternary ammonium compounds (quats) and iodine. These are the only three that have been approved by the Food and Drug Administration (FDA) (10). To achieve appropriate sanitization of tableware and utensils, an adequate amount of chemical must be added, but not too much - to avoid any toxicity effects (11).

\section{c. British Columbia Legis/ation}

The BC Food Premises regulation states that utensils used be maintained in a sanitary condition and be washed in a way that makes them free of contamination (1). However, it does not provide further explanation on how that should be achieved. The BC Food Retail and Food Services Code lists what the required sanitizer concentration of the approved chemicals needs to be at various temperatures (2). 
Table 1. Allowable minimum chlorine concentrations and temperature combinations for mechanical dishwashing (2).

\begin{tabular}{|ccc|}
\hline $\begin{array}{c}\text { MinimumConcentration } \\
\mathbf{m g} / \mathbf{l}(\mathbf{p p m})\end{array}$ & $\begin{array}{c}\text { Minimum Temperature } \\
\mathbf{p H ~} 8 \text { to } 10\end{array}$ & $\begin{array}{c}\text { Minimum Temperature } \\
\text { pH } 8 \text { or less }\end{array}$ \\
25 & $49^{\circ} \mathrm{C}\left(120^{\circ} \mathrm{F}\right)$ & $49^{\circ} \mathrm{C}\left(120^{\circ} \mathrm{F}\right)$ \\
50 & $38^{\circ} \mathrm{C}\left(100^{\circ} \mathrm{F}\right)$ & $24^{\circ} \mathrm{C}\left(75^{\circ} \mathrm{F}\right)$ \\
100 & $13^{\circ} \mathrm{C}\left(55^{\circ} \mathrm{F}\right)$ & $13^{\circ} \mathrm{C}\left(55^{\circ} \mathrm{F}\right)$ \\
\hline
\end{tabular}

\section{d. 5-log reduction of pathogenic microorganism}

There are approximately 21 million norovirus cases a year in the U.S, making it the major cause of epidemic gastroenteritis (11). Of the 21 million norovirus cases, 5.5 million are the result of foodborne illnesses (12). Feliciano (2012) measured the efficacies of sanitizers such as chlorine and quaternary ammonium compounds on reducing the load of viruses' compared to bacteria during warewashing (11). Bolton (2013) studied the virucidal efficacies of liquid sanitizers on viruses (12). Due to laboratory restrictions, the use of human norovirus (HuNoV) is not yet possible so a surrogate norovirus was used: Murine Norovirus (MNV-1) (12).

Feliciano tested three different contact surfaces - the most common ones used in a food services establishment - ceramic plates, drinking glasses and stainless steel cutlery. All three were soiled with food that was inoculated with murine norovirus (MNV-1), Escherichia coli K-12 and Listeria innocua. Her results indicated that regardless of the type of surface, chlorine at the appropriate concentrations was very effective at inactivating Escherichia coli K-12 and Listeria innocua, but not MNV-1 (11).

As mentioned earlier, sanitization of a food contact surface is meant to achieve a 5 -log reduction of most pathogenic microorganisms, with the correct amount of chemical sanitizer (10). However, Feliciano (2012) revealed that the 5 -log reduction only applied to bacteria and not viruses, which is contrary to what the legislation states and requires. The BC Food Premises Regulations define sanitization as the removal of microorganisms (1) . However, sound science states that viruses although acellular, are microorganisms (13). Since viruses are technically microorganisms, there should also be a $99.999 \%$ reduction of them during sanitization treatment process. Yet, the chemicals being used do not achieve such level of removal.

Bolton, on the other hand, compared virucidal efficacies of various liquid sanitizers. The three tested were $5 \%$ levulinic acid plus $2 \%$ SDS [LEV/SDS], 200 ppm chlorine, and an isopropanol-based quaternary ammonium compound [Alpet D2] (12).

The surface tested was stainless steel, though, the surface should not make a difference (11). Treatment was applied in three ways: "by conventional hydraulic or air-assisted, induction-charged (AAIC) electrostatic spraying or by wiping with impregnated towelettes" (12). It was concluded that hydraulic spraying was overall the best application to remove contaminants. Hydraulic spraying is the mechanism used in dishwashers. However, $M V N-1$ showed the highest removal when treated with LEV/SDS wipes or 200 ppm chlorine wipes over ALPET wipes. Mechanical action of the wipes may have been an assisting factor. It was also shown that removal of organic material before sanitizing plays a substantial role in reduction levels (12). Park (14) found that to receive only a 2 log reduction of MNV-1, a chlorine concentration of 5,000 ppm must be used - considerably above the standards. Feliciano (11) also suggested that viruses may be inactivated if the concentration of the sanitizer is increased, 
but to what extent is unclear. In the process of trying to remove a biological hazard we may instead introduce a chemical hazard.

\section{e. Applying knowledge from other public health sectors to a food service establishment}

Most often, the type of sanitizer selected when using a low temperature dishwasher is chlorine. Chlorine is the chemical of choice not only for warewashing operations, but also for treating drinking water and disinfecting recreational water and swimming pools. It is more popular than other disinfectants because of its affordability and capability of maintaining a residual during transport and storage, which protects the water from recontamination (15).

The goal of Murray's (15) experiment was to determine which method of testing chlorine levels in drinking water gave the most accurate results. A few tests were used, such as La Motte Colorimeter, LaMotte Test Tube Kit, Precision Laboratories Test Strips, Pentair Pool Test Kit, Hach Color Wheel Test Kit and Hach AquaChek Test Strips (15). These methods of testing water levels is not appropriate for warewashing, as these kits only test minute levels of chlorine: levels that are safe for ingestion. Volunteers found that using test strips was extremely easy but the results could not be presented with confidence. Whereas, the colorimeter was the opposite - hard to perform, but produced confident results (15). Although this experiment was conducted on drinking water, and not on a dishwasher's residual water or surface of a utensil, the same concept can be applied. Is the method that is currently being used to test the concentration sufficient? If so, should the method be standardized and written in legislation so that it is consistent each time? If it is not, what other method could be used that will be suitable for a dishwasher and utensils?

In another experiment conducted by Britta Brands (16), dishwasher hygiene was tested for domestic dishwashers that reuse water. Reusing water is a shrewd from an environmental protection standpoint, because it reduces the amount of water that a dishwasher uses. Water that is used for the last rinse of a load/cycle is recycled and used as the pre-rinse of the next cycle. This study examined whether or not more microbes were introduced into the dishwasher and the utensils when water was reused. It was concluded that microbial loads increase in all domestic dishwashers over time, due to the introduction of them when placing dirty dishes into the dishwasher. However, the ones that have a reservoir to hold the water used in the last cycle, did show a higher microbial concentration (16). Although there is a higher microbial concentration inside the dishwasher, it did not affect the cleanliness of the utensils. Tableware from both types of dishwashers produced the same level of microbial reduction after washing. Perhaps drying is a most crucial step in a dishwashing operation (16).

The knowledge attained from Murray and Brands' studies can be applied to commercial dishwashers. Brands revealed that the internal parts of dishwashers, depending on the brand and type, might be different, and therefore react differently to microbial growth. Thus it is more important to test the final object, which in our case would be the surface of the utensil, when measuring for adequate sanitation.

\section{f. Strengths and limitations of the literature review}

This review had several limitations; in particular, there was limited information pertaining to how warewashing operations 
should be assessed. This may be because washing utensils appears to be a fairly easy task, thus not getting the attention it requires. However, if not done correctly, it can have adverse health effects on the public. Another limitation of this review was the heavy reliance on product manufactures websites for information. Products manufactures are biased, and will tend to overvalue their products.

A strength of this review was that peerreviewed articles surrounding warewashing in food service establishments were used. These journals focused on other relatively important topics such as sanitizer concentrations required to destroy viruses on food contact surfaces, and which food residues shield pathogens the most, making it harder to achieve sanitation.

\section{g. Conclusion of Evidence Review}

The current literature published does not highlight the importance of measuring the residual concentration of a chemical after a dishwashing cycle. The food premises inspection system lacks a technique that EHOs can use in their routine inspections. Though the differences between the two methods currently used are not drastic, it would be worthy to formalize a technique for the purpose of consistency.

\section{Methods}

Chlorine test papers formally called "Chlorine Test Papers - Code 4250-BJ" from LaMotte Laboratories (17) were used to measure the concentration of the residual sanitizer concentration. A normal dishwasher cycle was run with the same dishes each time. The same number and type of dishes (ceramic bowls) were used for each run to keep the tests consistent and eliminate any factors that could influence the results. The chlorine test papers were dipped in two areas - the area of the ceramic bowl that had collected water and the exterior portion of the dishwasher that collected the rinse water. It was vital that the dishware was still wet when taking the reading because the test papers only react when in contact with a liquid.

Each dishwasher run provided sample data for both the dishware and the rinse water. The experiment was conducted over two days, with 15 samples per group collected each day. The tests were conduced at exactly the same times each day by the same researcher, to eliminate any external factors that could influence the results. The LaMotte Chlorine test papers, which are chemically treated paper strips, were used to determine the concentration of chlorine in ppm. The change in color from white to a certain purple (faint, light, medium or dark) indicated the concentration. The four distinct color changes occur at $10 \mathrm{ppm}, 50$ ppm, 100 ppm, and 200 ppm (17). For concentrations that fall between those ranges, there is no color standard to follow; therefore, the researcher determined what the concentration was, using sound judgment. The mean concentrations of the groups were then compared to see if there was any discrepancy between them. The temperature of the water was also taken before the first sample and after the last sample for each day. This was done to ensure that the temperature and chlorine concentration combinations were meeting the requirements from the guideline.

\section{Results}

\section{Descriptive Statistics}

In this study, numeric discrete data was collected. The residual sanitizer (chlorine) concentration was measured in parts per million (ppm). The mean residual concentration on the dishware was compared with the mean residual concentration on the rinse water. The 
descriptive statistics used in this study were the mean, range, minimum, and maximum.

Table 2. Descriptive statistics of Group Utensil and Group Rinse Water

\begin{tabular}{|c|c|c|c|c|}
\hline Group & $\mathbf{N}$ & $\begin{array}{c}\text { Mea } \\
\mathbf{n}\end{array}$ & $\begin{array}{c}\text { Minimu } \\
\mathbf{m}\end{array}$ & $\begin{array}{c}\text { Maximu } \\
\mathbf{m}\end{array}$ \\
\hline $\begin{array}{c}\text { Utensi } \\
\text { I }\end{array}$ & $\begin{array}{c}3 \\
0\end{array}$ & 86 & 60 & 110 \\
\hline $\begin{array}{c}\text { Rinse } \\
\text { Water }\end{array}$ & 3 & 93 & 70 & 110 \\
\hline
\end{tabular}

\section{Null and Alternative Hypotheses}

The hypotheses generated were as follows: $\mathbf{H}_{\mathbf{o}}$ : There is no difference in residual sanitizer concentration in the rinse water and at plate surface. $\left(\mu_{1}=\mu_{2}\right)$

$\mathbf{H}_{\mathbf{a}}$ : There is a difference in residual sanitizer concentration in the rinse water and at plate surface. $\left(\mu_{1} \neq \mu_{2}\right)$

\section{Inferential Statistics}

The statistical packaged used was SPSS (18). An independent samples t-test (9) was used to compare the two means of residual sanitizer concentration on the surface of the dishware and in the rinse water to assess whether there was a significant difference between the two groups. A twotailed test was used because prior to the study there was no knowledge as to which direction the mean difference would be.

The number of samples used for each group was 30. Group One (Dishware) had a mean concentration of $86 \mathrm{ppm}$ while Group Two (Rinse Water) had a mean concentration of $93 \mathrm{ppm}$. Since the data was normally distributed, the parametric test was read. From the Equal-Variance T-test, $p$ was equal to 0.035 therefore the null hypothesis was rejected and it was concluded that there is a statistically significant difference between the residual concentration at the rinse water and at the surface of the dishware.

\section{Discussion}

The purpose of this research study was to analyze the two locations that are often used to measure the residual concentration of a sanitizer used after a dishwasher cycle has been completed. Residual testing is important because it verifies that there was enough sanitizer in the dishwasher and that the dishwasher was doing its job. A dishwasher's job is to eliminate diseasecausing pathogens on dishes to a level that is safe from a public health standpoint. Levels that are safe for human consumption have already been pre-determined and sanitizer concentration levels have been set based on that standard. The Food Retail and Food Services (Food Code) (2) code indicates that the minimum chlorine concentration is 25,50 , or $100 \mathrm{ppm}$ depending on the temperature of the dishwasher water. The lower the water temperature, the higher the chlorine concentration required. For example, water that is 13 degrees Celsius would require a chlorine concentration of $100 \mathrm{ppm}$ to achieve levels of microbial reduction that is considered safe for human health. It is often up for debate as to which location is the correct one; this can sometimes cause discrepancy amongst Environmental Health Officers (EHOs) as well as with the operators and staff of a food service establishment. One location that is used is the area where the rinse water is collected, and the other location is directly on the dish, usually the concaved end of the dish where it able to collect some water. Water must be present on the dish in order for the test strip to work correctly. Although the Food Retail and Food Services Code (Food Code) (2) and the BC Food Premises Regulations (Regulations) (1) state what the residual concentrations should be, there is 
no universal method stated on how to test for it. The results from this study demonstrated that the two locations that are most often used, do have a statistically significant difference. Following the analysis of the inferential statistics (18), the author was able to conclude that the water on the surface of the plate had a lower mean residual than the rinse water. The following means were calculated for a dishwasher that used chlorine as the sanitizing chemical:

1. Water on the surface of the plate: $86 \mathrm{ppm}$

2. Rinse water: $93 \mathrm{ppm}$

The average temperature of the dishwasher during these cycles was 50 degrees Celsius. According to the Food Code, a water temperature of 49 degrees Celsius only needs a chlorine concentration of $25 \mathrm{ppm}$ (2). In this study, all the dishwasher cycles were meeting the required temperature and sanitizer concentration requirements. While the findings only suggest a concentration difference of seven ppm between the two locations, it is still sufficient to suggest that a standardized method may be beneficial. It will be beneficial primarily because it produces consistency amongst EHOs and the staff of the food service establishment, thus lending credence to what an EHO says during an inspection. The public health significance of measuring residual concentration is that dishes and utensils are food contact surfaces, and should always be free of pathogens to prevent the spread of communicable diseases. If sanitized adequately, such that at the correct sanitizer concentrations and correct temperatures, then it can be assumed that disease-causing microorganisms are suppressed to levels that are safe for human consumption.

It is important to keep in mind that with a low temperature dishwasher, the sanitizer concentration is not the only thing that needs to be tested for. Chemical dishwashers also have minimum and maximum allowable temperatures. Provided that these dishwashers use a chemical sanitizer to do the work of eliminating pathogens, the temperature requirements are often low, i.e., between $13^{\circ} \mathrm{C}$ and $49^{\circ} \mathrm{C}(2)(6)$, and thus this is usually in compliance. It is also vital that the temperatures do not exceed the maximum allowable value of $54{ }^{\circ} \mathrm{C}(6)$, as this can create some corrosive effects.

The different results from the two locations do not suggest that a change in legislation is required immediately to enhance public health. Instead, these results suggest that an adjustment in the way assessments are currently conducted may lead to a better understanding between those working in public health and also those in the food service industry. In the end, this will only lead to more public health protection, especially among patrons of a food service establishment.

\section{Limitations}

One of the limitations of this study was that it was carried out in a single food service establishment and only one type of dishwasher was used. Although these results concluded that the two different areas produce dissimilar results, it is not possible to say that this applies to all low temperature dishwashers. There are multiple brands and styles of low temperature dishwashers, and it was not feasible to test them all.

Another limitation of this study was that it only analyzed the chlorine residual, and not the other two officially accepted sanitizers: quaternary ammonium compounds (QUATS) and iodine. Although chlorine, QUATS and iodine are all used for sanitation because they all achieve the same end results, they also have very distinct properties of their own. From this study alone, it cannot be concluded that QUATS 
or iodine sanitizer residuals will be behave in a similar manner.

One of the greatest limitations of this study was the subjectivity of the results. The concentration was determined based on the strength of the color and decided upon the researcher's judgment. The LaMotte Chlorine test strips only have four color strengths to refer to when determining the concentration. The broadness of the ranges makes it difficult to give anything that falls between those values an exact numeric value. However, this limitation gives insight to how operators and EHOs conduct this test: most likely in a very similar and thus limited manner.

\section{Future Research}

Some suggestions for potential future studies are:

1. Compare the mean residual concentration for the two other sanitizers: QUATS and iodine.

2. Conduct this test on multiple brands and types of low temperature chemical dishwashers.

3. Test the residual concentration on different types of surfaces such as plastic, glass or stainless steel.

\section{Conclusions/ Recommendations}

In this study, the most frequently used method to measure residual sanitizer concentration by EHOs was identified and examined. Test papers are a simple method to measure residual concentration but are also subject to limitations. The limitations

\section{References}

1. Food Premises Regulation [Internet]. Canada; Available from: http://www.bclaws.ca/EPLibraries/b discussed earlier can affect the accuracy of the results. Nonetheless, this research was not carried out to determine if restaurants were meeting the required concentration requirements; instead, the study was conducted to determine if the two locations for measuring residual concentration had inconsistent results. Results from both testing locations were within an acceptable concentration; the minimum desirable concentration $50 \mathrm{ppm}$, which was observed for both test locations. From the information obtained from the tests, it was ascertained that it is best to measure the residual concentration at the wet surface of the dishware for two practical reasons.

First, it is always better to err on the side of caution; it is safer to assume that a low concentration is being dispensed, and increase the dose, with the assumption that it will not go over $200 \mathrm{ppm}$, rather than assuming a high concentration, and decreasing the dose, which can result in inadequate sanitation. Secondly, dishware is a food contact surface, while the rinse water is not; therefore, it only makes logical sense to test the residual on the dishware. Dishware is a vector capable of transmitting disease causing microorganisms if not sanitized adequately.

\section{Acknowledgements}

The authors thank the British Columbia Institute of Technology, Environmental Health department for supporting their research.

\section{Competing Interest}

The authors declare that they have no competing interests claws_new/document/ID/freeside/1 1_210_99

2. Canadian Food Inspection System Implementation Group. Food Retail and Food Services Code. 
2004;(September):1-89.

3. Schmidt RH. Basic elements of equipment cleaning and sanitizing in food processing and handling operations. 1997;

4. EcoLab [Internet]. [cited 2016 Oct 22]. Available from:

https://foodsafety.ecolab.com/us/fo od-safety/test-strips

5. Gibson KE, Almeida G. Comparison of a continuous flow dipper well and a reduced water dipper well combined with ultraviolet radiation for control of microbial contamination. Food Control. 2015;47:301-5.

6. Keener K. Cleaning and Sanitizing Operations. Microbiol Safe Foods. 2008;415-33.

7. Krog AJ, Dougherty DS. Effectiveness of the Methods of Dish and Utensil Washing in Public Eating and Drinking Establishments. Am J Public Health Nations Health [Internet]. 1936;26(9):897-900. Available from: http://www.ncbi.nlm.nih.gov/pubm ed/18014497\%0Ahttp://www.pubm edcentral.nih.gov/articlerender.fcgi? artid=PMC1562984

8. Macleod M. The Traditional Inspection. Burnaby; 2016.

9. Hobart Canada [Internet]. [cited 2016 Oct 22]. Available from: http://www.hobart.ca/

10. Gaulin C, Lê $M$, Shum M, Fong D. Disinfectants and sanitizers for use on food contact surfaces. Natl Collab Cent Environ Heal [Internet]. 2011;(August):1-15. Available from: http://www.ncceh.ca/sites/default/f
iles/Food_Contact_Surface_Sanitizer s_Aug_2011.pdf

11. Feliciano L, Li J, Lee J, Pascall MA. Efficacies of Sodium Hypochlorite and Quaternary Ammonium Sanitizers for Reduction of Norovirus and Selected Bacteria during WareWashing Operations. PLoS One. 2012;7(12).

12. Bolton SL, Kotwal G, Harrison MA, Law SE, Harrison JA, Cannon JL. Sanitizer efficacy against murine norovirus, a surrogate for human norovirus, on stainless steel surfaces when using three application methods. Appl Environ Microbiol. 2013;79(4):1368-77.

13. Levinson WE. Review of Medical Microbiology and Immunology. Lange Basic Sci [Internet]. 2008;1919:1147-72. Available from: http://web.b.ebscohost.com/ehost/ pdfviewer/pdfviewer?vid=20\&sid=ba 7801ec-d0b3-4ea3-824c-

6be2a134b281@sessionmgr110\&hid =119\%5Cnhttp://www.ncbi.nlm.nih. gov/pubmed/23618829\%5Cnhttp:// www.ncbi.nlm.nih.gov/pubmed/128 61227

14. Park GW, Barclay L, Macinga $D$, Charbonneau $D$, Pettigrew $C$ a, Vinjé

J. Comparative efficacy of seven hand sanitizers against murine norovirus, feline calicivirus, and GII.4 norovirus. J Food Prot. 2010;73(12):2232-8.

15. Murray A, Lantagne D. Accuracy, precision, usability, and cost of free chlorine residual testing methods. $J$ Water Health. 2015;13(1):79-90.

16. Brands B, Bockmühl DP. Experimental evaluation of hygienic 
conditions in domestic dishwashers.

Tenside, Surfactants, Deterg.

2015;52(2):148-54.

17. LaMotte. LaMotte Company

[Internet]. [cited 2016 Nov 20].

Available from:

http://www.lamotte.com/en/

18. IBM. IBM SPSS Statistics. Armonk:

IBM; 2015. 